\title{
An optimization approach to weak approximation of Lévy-driven stochastic differential equations
}

Kenji Kashima and Reiichiro Kawai

\begin{abstract}
We propose an optimization approach to weak approximation of Lévydriven stochastic differential equations. We employ a mathematical programming framework to obtain numerically upper and lower bound estimates of the target expectation, where the optimization procedure ends up with a polynomial programming problem. An advantage of our approach is that all we need is a closed form of the Lévy measure, not the exact simulation knowledge of the increments or of a shot noise representation for the time discretization approximation. We also investigate methods for approximation at some different intermediate time points simultaneously.
\end{abstract}

\section{Preface}

$\mathrm{I}^{1}$ have received my Bachelor, Master and Ph.D. degrees from Kyoto University under the supervision of Yamamoto-Sensei. As far as I know, my Bachelor thesis was the first trial to apply " $H^{\infty}$ signal reconstruction via sampled-data control theory" to actual audio data processing. I was extremely impressed to see the transdisiplinary aspect of system control theory. This invaluable experience is a source of my desire to contribute to other fields outside of the control community. Fortunately, I became acquainted with the second author whose speciality is probability theory and mathematical finance. In this article, we present our ongoing joint work that aspires to be as successful in the mathematical finance community as the YY-filter is in the signal processing world.

Kenji Kashima

Tokyo Institute of Technology, 2-12-1, Oh-okayama, Meguro-ku, Tokyo 152-8552, Japan, e-mail: kashima@mei.titech.ac.jp

Reiichiro Kawai

University of Leicester, Leicester LE1 7RH, UK. e-mail: reiichiro.kawai@gmail.com

${ }^{1}$ In this section, "I" refers to the first author, Kenji Kashima. 


\section{Introduction}

Stochastic differential equations have long been used to build realistic models in economics, finance, biology, the social sciences, chemistry, physics and other fields. In most active fields of application, dynamics with possible sudden shift have become more and more important. To model such shifts, one would like to employ stochastic differential equations where the underlying randomness contains jumps. For this purpose, the diffusion process is not sufficient since its sample paths are almost surely continuous. On the other hand, Lévy-driven stochastic differential equations, which contain diffusion as a special case, can formulate stochastic behavior with jumps. Regardless of its practical importance, however, the theory and the computational techniques of the Lévy processes have not been developed thoroughly as in the diffusion case. As nice references on the subject, we refer to Applebaum [1].

From a practical point of view, the sample paths approximation of stochastic differential equations has been a central issue for the purpose of numerical evaluation and simulation on the computer. There are two notions of the approximation; strong and weak approximations. The strong approximation schemes provide pathwise approximations which can be employed in scenario analysis, filtering or hedge simulation. For applications such as derivative pricing, the computation of moments or expected utilities, the so-called weak approximations are sufficient, that is, we need to estimate the expected value of a function. Other applications of the weak approximation include the computation of functional integrals, invariant measures, and Lyapunov exponents.

The theoretical properties of time discretization schemes are mostly studied for the diffusion case. See [6] for detailed investigation. In fact, the weak approximation of the Lévy-driven stochastic differential equations via Monte Carlo type methods is still very difficult. Moreover, the other existing methods are applicable only to some of the simplest Lévy processes. The main purpose of this paper is to propose a new approach to weak approximation of Lévy-driven stochastic differential equations. Unlike Monte Carlo simulation with the time discretization approximation of sample paths, we employ a mathematical programming framework to obtain numerically upper and lower bounds of the target expectation.

To this end, we follow the methodologies investigated in various fields of application by several authors, for example, Bertsimas, Popescu and Sethuraman [2], Helmes, Röhl and Stockbridge [4], Lasserre, Prieto-Rumeau and Zervos [8], to mention just a few. ${ }^{2}$. Note that these results deal only with the pure diffusion case (i.e., without jump component) for which standard Monte Carlo methods are sufficient. In this sense, it should be emphasized that our result is not a trivial extension. The main drawback is the complexity of the Ito formula for Lévy-driven stochastic differential equations. As such, we need to carefully examine whether or not the resulting opti-

\footnotetext{
${ }^{2}$ It is known that there exist two dual formulation of this framework, both of which arrive at a semidefinite programming in the end. One is the so-called generalized moment problem that makes use of the semi-definiteness of (localizing) moment matrices. The other is a polynomial optimization approach for which sum-of-squares relaxation efficiently works. In this paper, our discussion is based on the latter formulation.
} 
mization problems are practically solvable. Fortunately, as we show in the following sections, our approach covers various practically important Lévy-driven stochastic differential equations.

The rest of this paper is organized as follows. Section 2 gives mathematical definition of Lévy-driven stochastic differential equations. Section 3 introduces and studies our optimization approach to the weak approximation. Section 4 provides a numerical example to illustrate that our method is able to efficiently capture the marginal distributions of Lévy-driven stochastic differential equations. Finally, Section 5 concludes this paper.

\section{Problem formulation}

Let us begin this section with general notations which will be used throughout the text. For $k \in \mathbb{N}, \partial_{k}$ indicates the partial derivative with respect to $k$-th argument. We denote by $C^{k_{1}, k_{2}}$ the class of continuous functions with continuous differentiability of $k_{1}$-time for the first argument and of $k_{2}$-time for the second argument.

Let $X_{0}$ be given in $\mathbb{R}$ and let $T>0$. Consider a one-dimensional stochastic differential equation

$$
d X_{t}=a_{0}\left(t, X_{t}\right) d t+a_{1}\left(t, X_{t}\right) d W_{t}+\int_{\mathbb{R}_{0}} b\left(t, X_{t-}, z\right)(\mu-v)(d z, d t), \quad t \in[0, T],
$$

where $\left\{W_{t}: t \geq 0\right\}$ is a standard Brownian motion and where $\mu$ is a Poisson random measure on $\mathbb{R}_{0}$ whose compensator is given by the Lévy measure $v$ satisfying $\int_{|z|>1}|z| v(d z)<+\infty$ and $\int_{\mathbb{R}_{0}}\left(|z|^{2} \wedge 1\right) v(d z)<+\infty$. In order for the solution of (1) to be well defined, we impose the usual Lipschitz conditions and linear growth conditions on $a_{0}, a_{1}$ and $b$. We henceforth equip our underlying probability space with the natural filtration $\left(\mathscr{F}_{t}\right)_{t \in[0, T]}$ generated by $\left\{X_{t}: t \in[0, T]\right\}$. Moreover, throughout this study, we assume that $b(t, x, z) \neq 0$ and $v \neq 0$ to avoid triviality.

Our interest throughout this study is in approximating the expectation

$$
\mathbb{E}\left[V\left(X_{T}\right)\right] .
$$

Here, $V$ is a function mapping from $[0, T] \times \mathbb{R}$ to $\mathbb{R}$, piecewise polynomial in $t$ and $x$ and such that $\mathbb{E}\left[\left|V\left(X_{T}\right)\right|\right]<+\infty$. Note that the function $V$ may have discontinuities. For the computation of $\mathbb{E}\left[V\left(X_{T}\right)\right]$, standard techniques include the Monte Carlo simulation of sample paths through the time discretization of stochastic differential equations, or even some exact knowledge of sample paths such as series representation of the Poisson jump component. 


\section{Optimization approach to weak approximation}

\subsection{Ito formula and supermartingale}

We are now in a position to introduce our optimization approach to the weak approximation. Let $\mathscr{X}(\subseteq \mathbb{R})$ be a support of $\left\{X_{t}: t \in[0, T]\right\}$ defined in (1). For $f \in C^{1,2}([0, T] \times \mathscr{X} ; \mathbb{R})$, the Ito formula yields

$$
\begin{aligned}
d f\left(t, X_{t}\right)= & \mathscr{A} f\left(t, X_{t}\right) d t+\partial_{2} f\left(t, X_{t}\right) a_{1}\left(X_{t}\right) d W_{t} \\
& +\int_{\mathbb{R}_{0}} B_{z} f\left(t, X_{t-}\right)(\mu-v)(d z, d t), \quad \text { a.s. }
\end{aligned}
$$

where

$$
\begin{aligned}
\mathscr{A} f(t, x):= & \partial_{1} f(t, x)+\partial_{2} f(t, x) a_{0}(x)+\frac{1}{2} \partial_{2}^{2} f(t, x) a_{1}(x)^{2} \\
& +\int_{\mathbb{R}_{0}}\left(B_{z} f(t, x)-\partial_{2} f(t, x) b(x, z)\right) v(d z) .
\end{aligned}
$$

and for $z \in \mathbb{R}_{0}$,

$$
B_{z} f(t, x):=f(t, x+b(x, z))-f(t, x) .
$$

Here, if

$$
\mathbb{E}\left[\int_{0}^{T}\left(\partial_{2} f\left(t, X_{t}\right) a_{1}\left(t, X_{t}\right)\right)^{2} d t\right]<+\infty
$$

and if

$$
\mathbb{E}\left[\int_{0}^{T} \int_{\mathbb{R}_{0}}\left(B_{z} f\left(t, X_{t}\right) a_{1}\left(t, X_{t}\right)\right)^{2} v(d z) d t\right]<+\infty,
$$

then the stochastic process

$$
\left\{f\left(t, X_{t}\right)-f\left(0, X_{0}\right)-\int_{0}^{t} \mathscr{A} f\left(s, X_{s}\right) d s: t \in[0, T]\right\}
$$

is a square-integrable martingale with respect to the filtration. We can then derive one of important building blocks of our approach, the so-called Dynkin formula:

$$
\mathbb{E}\left[f\left(T, X_{T}\right)\right]-f\left(0, X_{0}\right)=\mathbb{E}\left[\int_{0}^{T} \mathscr{A} f\left(s, X_{s}\right) d s\right] .
$$

Hence, as soon as one finds an $f \in C^{1,2}([0, T] \times \mathscr{X} ; \mathbb{R})$ such that

$$
\left\{\begin{aligned}
\mathscr{A} f(t, x) & \leq 0,(t, x) \in[0, T] \times \mathscr{X} \\
f(t, x) & \geq V(x), x \in \mathscr{X}
\end{aligned}\right.
$$

it follows

$$
\mathbb{E}\left[V\left(X_{T}\right)\right] \leq \mathbb{E}\left[f\left(T, X_{T}\right)\right] \leq f\left(0, X_{0}\right) .
$$


Clearly, $f\left(0, X_{0}\right)$ serves as an upper bound of $\mathbb{E}\left[V\left(X_{T}\right)\right]$. To minimize the upper bound $f\left(0, X_{0}\right)$, we now turn to the optimization problem

$$
\begin{array}{ll}
\min & f\left(0, X_{0}\right) \\
\text { s.t. } & f(t, x) \geq V(x), x \in \mathscr{X} \\
& \mathscr{A} f(t, x) \leq 0,(t, x) \in[0, T] \times \mathscr{X}, \\
& f \in C^{1,2}([0, T] \times \mathscr{X} ; \mathbb{R}) .
\end{array}
$$

\subsection{Main result}

This optimization problem is very difficult to deal with since the class definitions of the functions $f$ and $V$ are too broad. To ease the above optimization problem, we restrict the class of the function $f$ to be a polynomial both in $t$ and $x$, that is, in the form

$$
f(t, x)=\sum_{\left\{0 \leq k_{1} \leq K_{1}, 0 \leq k_{2} \leq K_{2}\right\}} c_{k_{1}, k_{2}} t^{k_{1}} x^{k_{2}},
$$

for some natural numbers $K_{1}$ and $K_{2}$ and for a sequence $\left\{c_{k_{1}, k_{2}}\right\}_{k_{1} \leq K_{1}, k_{2} \leq K_{2}}$ of constants. For convenience in notation, we henceforth denote by $C_{p}$ the class of polynomial functions in the form (6). We also need to set $V$ to be a piecewise polynomial both in $t$ and $x$. Moreover, we assume that both $a_{0}$ and $a_{1}$ are polynomials. We are then instead to solve the following optimization problem

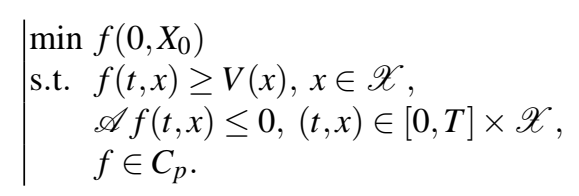

For the purpose of comparison, suppose that there is no jump in (1), that is, $b \equiv 0$ as in [11]. This assumption clearly makes $\mathscr{A} f$ a polynomial, and consequently (7) is a polynomial optimization problem. This is the main reason that the pure diffusion case is easier to deal with in this framework. In general, polynomial optimization problems are still NP hard. However, if the degrees of $f$ are fixed, sums of squares relaxation enables us to solve the problem efficiently. For details, we refer to Parrilo [9]. On the other hand, this technique is not directly applicable to the model with general stochastic jumps. This is because $\mathscr{A} f$ is not necessarily a polynomial due to the additional integral term.

To circumvent this difficulty, we decompose the function $b$ as follows:

Assumption 1 Functions $a_{0}$ and $a_{1}$ are polynomials, and $b$ is decomposed as

$$
b(t, x, z)=b_{1}(t, x) b_{2}(z)
$$

where $b_{1}$ is a polynomial in $x$ and where $b_{2}: \mathbb{R}_{0} \mapsto \mathbb{R}$ such that 


$$
\int_{\mathbb{R}_{0}}\left|b_{2}(z)\right|^{k} v(d z)<+\infty, \quad k=2, \ldots, K_{2} .
$$

Theorem 1. Under Assumption 1, for any $f \in C_{p}, \mathscr{A} f$ is a polynomial in $t$ and $x$. Moreover, the coefficients of $\mathscr{A} f$ are affine with respect to those of $f$.

Proof: A simple algebra yields

$$
\begin{aligned}
\mathscr{A} f(t, x)= & \partial_{1} f(t, x)+\partial_{2} f(t, x) a_{0}(t, x)+\frac{1}{2} \partial_{2}^{2} f(t, x) a_{1}(t, x)^{2} \\
& +\sum_{\left\{0 \leq k_{1} \leq K_{1}, 2 \leq k_{2} \leq K_{2}\right\}} c_{k_{1}, k_{2}} t^{k_{1}} \sum_{k=0}^{k_{2}-2} k_{2} C_{k} x^{k} b_{1}(t, x)^{k_{2}-k} M_{k_{2}-k}
\end{aligned}
$$

where

$$
M_{l}:=\int_{\mathbb{R}_{0}} b_{2}(z)^{l} v(d z), l=2, \ldots, K_{2} .
$$

This completes the proof.

Clearly, the optimization (7) is now a polynomial programming problem. To be more precise, this problem is numerically tractable for any piecewise polynomial $V$. Finally, to obtain a lower bound for $\mathbb{E}\left[V\left(X_{T}\right)\right]$, we are to find a $g \in C_{p}$ via the polynomial programming

$$
\begin{array}{ll}
\max & g\left(0, X_{0}\right) \\
\text { s.t. } & g(t, x) \leq V(x), x \in \mathscr{X} \\
& \mathscr{A} g(t, x) \geq 0,(t, x) \in[0, T] \times \mathscr{X}, \\
& g \in C_{p} .
\end{array}
$$

Notice that our optimization approach does not require the sample paths simulation at all for the computation of the expectation $\mathbb{E}\left[V\left(X_{T}\right)\right]$. It is a great advantage of our approach that all we need is the Lévy measure in closed form, not the exact knowledge of the increments or of a shot noise representation for sample paths simulation for the weak approximation with the sample paths discretization.

\subsection{Simultaneous approximation for homogeneous process}

In this section, we show that the optimal solution obtained through our approach provides some additional information, that are of direct practical use.

Firstly, note that the initial value $X_{0}$ does not appear in the constraints (4) in the previous section. Therefore, if $f$ satisfies (4), $f(0, \tilde{x})$ automatically gives upper bounds for $\mathbb{E}_{\tilde{x}}\left[V\left(X_{T}\right)\right]$, where the notation $\mathbb{E}_{x}$ denotes the expectation taken under which the initial state of the stochastic differential equation (1) is given deterministically by $X_{0}=x$. 
The next theorem indicates that functions satisfying (4) can also serve as bounds at arbitrary intermediate time points.

Theorem 2. Assume that (1) is time-homogeneous, i.e., $a_{1}, a_{2}$, and $b$ are independent of $t$. Suppose that $f \in C^{1,2}$ satisfies (4). Then, for every $\bar{T} \in[0, T]$

$$
\mathbb{E}\left[V\left(X_{\bar{T}}\right)\right] \leq f\left(T-\bar{T}, X_{0}\right)
$$

Proof: Define

$$
f^{\circ}(t, x):=f(t+(T-\bar{T}), x) .
$$

Due to the time homogeneity, we have

$$
\mathscr{A} f^{\circ}(t, x)=\mathscr{A} f(t+(T-\bar{T}), x) \leq 0,(t, x) \in[0, \bar{T}] \times \mathscr{X} .
$$

We also have

$$
f^{\circ}(\bar{T}, x)=f(T, x) \leq V(x), \text { in } \mathscr{X} .
$$

By combining these inequalities and Dynkin formula, we obtain

$$
\begin{aligned}
\mathbb{E}\left[V\left(X_{\bar{T}}\right)\right] & \leq \mathbb{E}\left[f^{\circ}\left(\bar{T}, X_{\bar{T}}\right)\right] \\
& =f^{\circ}\left(0, X_{0}\right)+\mathbb{E}\left[\int_{0}^{\bar{T}} \mathscr{A} f^{\circ}\left(s, X_{s}\right) d s\right] \\
& \leq f\left(T-\bar{T}, X_{0}\right) .
\end{aligned}
$$

This completes the proof.

We here make a brief comment on the choice of the cost function in the optimization problem. When we attempt to as tight bounds for (1) as possible, we should solve (7) and (8). However, we need to approximate $V\left(X_{\bar{T}}\right)$ for some different time points $\bar{T} \in[0, T]$ and also different initial value $X_{0}$, it is useful to suitably change the cost function. Fortunately, for suitable measure $\phi$ on $[0, T] \times \mathbb{R}$, we can similarly optimize

$$
\int f(t, s) \phi(d t, d s), \int g(t, s) \phi(d t, d s)
$$

since these are linear combination of the decision variables (the coefficients of $f$ and $g$ ).

\section{Numerical Examples}

In this section we give some approximation examples. In the numerical examples presented hereafter, we utilize MATLAB SOSTOOLS combined with SeDuMi [10, $14]$, using a computer with a Pentium $43.2 \mathrm{GHz}$ processor and $2 \mathrm{~GB}$ memory. 


\subsection{Ornstein-Uhlenbeck-type process with gamma stationary distribution}

Let $v$ be a Lévy measure on $\mathbb{R}_{+}$such that $\int_{\mathbb{R}_{+}} z v(d z)<+\infty$. Set $a_{0}(t, x)=-\lambda x+$ $\int_{\mathbb{R}_{+}} z v(d z)$ for some $\lambda>0, a_{1}(t, x)=0, b_{1}(t, x)=1, b_{2}(z)=z$, and $X_{0}$ is independent of $\mu$. Then, the stochastic differential equation (1) reduces to

$$
d X_{t}=-\lambda X_{t} d t+\int_{\mathbb{R}_{+}} z \mu(d z, d t)
$$

which is called an Ornstein-Uhlenbeck-type process. (See, for example, Sato [13] for its details.) Its solution is given by

$$
X_{t}=e^{-\lambda t} X_{0}+\int_{0}^{t} \int_{\mathbb{R}_{+}} e^{-\lambda(t-s)} z \mu(d z, d s) .
$$

For simplicity, we further fix $X_{0}=0, \lambda=1$ and $v(d z)=b a e^{-b z} d z$, where $a>0$ and $b>0$. Then, we can prove that the stationary distribution of $\left\{X_{t}: t \geq 0\right\}$ is gamma with density $p(x)=b^{a} / \Gamma(a) x^{a-1} e^{-b x}, x \in \mathbb{R}_{+}$.

Here, we investigate the distribution transition via the moment estimations of $\mathbb{E}\left[X_{t}\right]=\left(1-e^{-t}\right) a / b, \mathbb{E}\left[X_{t}^{2}\right]=\left(1-e^{-2 t}\right) a / b^{2}+\left(1-e^{-t}\right)^{2} a^{2} / b^{2}$, and $\lim _{t \uparrow+\infty} \mathbb{E}\left[X_{t}^{k}\right]=$ $\Gamma(a+k) /\left(b^{k} \Gamma(a)\right)$, for $k \in \mathbb{N}$. Note that $\mathscr{X}=\mathbb{R}_{+}$and that $\int_{\mathbb{R}_{+}} z^{k} v(d z)=a k ! / b^{k}$ for $k \in \mathbb{N}$. For $f \in C_{p}\left([0, T] \times \mathbb{R}_{+} ; \mathbb{R}\right)$, we have

$$
\begin{aligned}
\mathscr{A} f(t, x)= & \sum_{\left\{1 \leq k_{1} \leq K_{1}, 0 \leq k_{2} \leq K_{2}\right\}} c_{k_{1}, k_{2}} k_{1} t^{k_{1}-1} x^{k_{2}} \\
& +\left(-x+\frac{a}{b}\right) \sum_{\left\{0 \leq k_{1} \leq K_{1}, 1 \leq k_{2} \leq K_{2}\right\}} c_{k_{1}, k_{2}} t^{k_{1}} k_{2} x^{k_{2}-1} \\
& +\sum_{\left\{0 \leq k_{1} \leq K_{1}, 2 \leq k_{2} \leq K_{2}\right\}} c_{k_{1}, k_{2}} t^{k_{1}} \sum_{k=0}^{k_{2}-2}{ }_{k_{2}} C_{k} x^{k} \frac{a\left(k_{2}-k\right) !}{b^{k_{2}-k}} .
\end{aligned}
$$

The condition (2) holds for each $K_{1}$ and $K_{2}$, since

$$
\int_{0}^{t} \int_{\mathbb{R}_{+}} e^{-\lambda(t-s)} z \mu(d z, d s) \leq \int_{0}^{T} \int_{\mathbb{R}_{+}} z \mu(d z, d s), \quad \text { a.s. }
$$

where the right hand side is an infinitely divisible random variable, whose Lévy measure has an exponential decay at infinity.

We present numerical results in Table 1 . We set $K_{1}=p$ for the estimation of the $p$-th moment. It is known that the computational burden for solving the polynomial optimization via sum of squares decomposition significantly increases as the degree of the polynomial becomes larger. In view of this, we choose large $K_{2}=10$. Even in this case, however, computation time is at most 2 seconds. For comparison with Monte Carlo methods, we also provide 99\%-confidence interval with 1000000 iid samples. As can be observed, even with the extraordinarily large number of samples, 
the 99\%-confidence intervals are far from being comparable with our results. Note that any large sample size can never be in competition with our results since the upper and lower bounds obtained through our method form nothing but the $100 \%$ confidence interval.

\begin{tabular}{c||c|c|c||c} 
& $t=1$ & $t=2$ & $t=3$ & $t \uparrow+\infty$ \\
\hline \hline \multirow{2}{*}{$\mathbb{E}\left[X_{t}\right]$} & $0.042141-0.042141$ & $0.057644-0.057644$ & $0.063347-0.063348$ & \\
& $(0.042141)$ & $(0.057644)$ & $(0.063348)$ & $(0.06667)$ \\
& {$[0.0417327,0.0427467]$} & {$[0.0569853,0.0580576]$} & {$[0.0628788,0.0639666]$} & \\
\hline \multirow{2}{*}{$\mathbb{E}\left[X_{t}^{2}\right]$} & $0.040205-0.040205$ & $0.046952-0.046955$ & $0.048331-0.048347$ & \\
& $(0.040205)$ & $(0.046953)$ & $(0.048347)$ & $(0.04889)$ \\
\hline \multirow{2}{*}{$\mathbb{E}\left[X_{t}^{3}\right]$} & {$[0.0396179,0.0414835]$} & {$[0.0457206,0.0476003]$} & {$[0.0476659,0.0496044]$} & \\
& $0.061217-0.061268$ & $0.066812-0.066886$ & $0.068009-0.068051$ & \\
$(\mathrm{n} / \mathrm{a})$ & $(\mathrm{n} / \mathrm{a})$ & $(\mathrm{n} / \mathrm{a})$ & $(0.06844)$ \\
& {$[0.0591606,0.0649658]$} & {$[0.0633623,0.0688394]$} & {$[0.0660050,0.0719257]$} &
\end{tabular}

Table 1 Moment transition with $X_{0}=0$ and $(a, b)=(0.1,1.5)$. The numbers in parentheses indicate theoretical value. The intervals are $99 \%$-confidence interval with 1000000 independent samples.

Recall that the current model is time-homogeneous. Hence, according to Theorem 2, the obtained bounding functions also give upper and lower bounds for intermediate time points without solving other optimization problem. For example, as a byproduct of the computation of the bounds for $\mathbb{E}\left[X_{3}^{3}\right]$, we can provide a parametric bounds for $\mathbb{E}\left[X_{t}^{3}\right]$ for every $t \in[0,3]$; see Fig. 1 . In this case, the accuracy is close to the pointwise optimization result in Table 1. Actually, the gap is smaller than the case of Monte Carlo methods in Table 1.

Fig. 1 Lower and upper bounds for $\mathbb{E}\left[X_{t}^{3}\right]$ at intermediate time points $t \in[0,3]$. The detailed values are $0.060992-0.061467(t=1)$ and $0.066461-0.067219$ $(t=2)$.

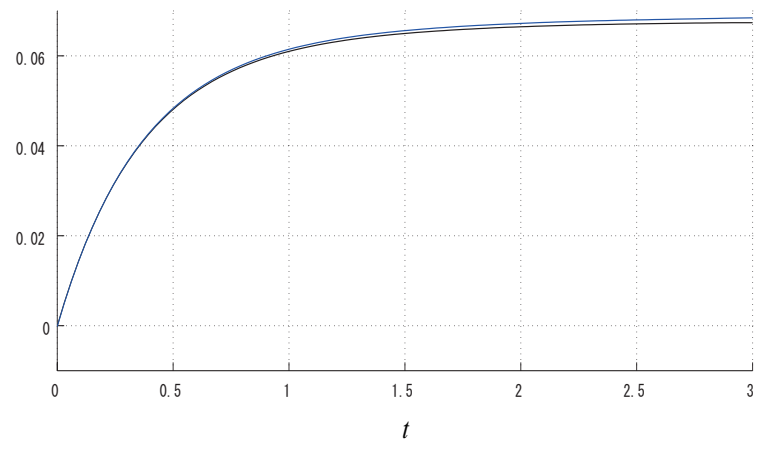




\section{Conclusion}

In this paper, we have developed a new approach to the weak approximation of Lévy-driven stochastic differential equations via an optimization problem yielding upper and lower bounds on the target expectation. The advantage of our approach is that all we need is the Lévy measure in closed form. We need neither the exact knowledge of the increments nor a shot noise representation for sample path simulation for the weak approximation with the sample path discretization. We have also investigated how we can obtain accurate approximation at transient times.

The most important remaining work is the improvement of the approximation accuracy. It is a good direction to pursue to use exponentially tempered polynomials [5]. Other currently ongoing work is

- application to calibration in finance,

- construction of finite-horizon control theory for systems described by Lévy driven stochastic differential equations.

\section{References}

1. Applebaum, D. (2004) Lévy Processes and Stochastic Calculus, Cambridge University Press.

2. Bertsimas, D., Popescu, I., Sethuraman, J. (2000) Moment problems and semidefinite programming, In: Handbook on semidefinite programming: Theory, Algorithms, and Applications, H. Wolkovitz, ed., 469-509.

3. Eriksson, B., Pistorius, M. (2008) A method of moments approach to pricing double barrier contracts driven by a general class of jump diffusions, available at arXiv:0812.4548v1.

4. Helmes, K., Röhl, S., Stockbridge, R.H. (2001) Computing moments of the exit time distribution for Markov processes by linear programming, Operations Research, 49(4) 516-530.

5. Kashima, K., Kawai, R., (2009) A weak approximation of stochastic differential equations with jumps through tempered polynomial programming, submitted.

6. Kloeden, P.E., Platen, E. (1999) Numerical Solution of Stochastic Differential Equations, Third Printing, Springer, Berlin.

7. Lasserre, J.B., Prieto-Rumeau, T. (2004) SDP vs. LP relaxations for the moment approach in some performance evaluation problems, Stochastic Models, 20(4) 439-456.

8. Lasserre, J.B., Prieto-Rumeau, T., Zervos, M. (2006) Pricing a class of exotic via moments and SDP relaxations, Mathematical Finance, 16(3) 469-494.

9. Parrilo, P.A. (2003) Semidefinite programming relaxations for semialgebraic problems, Mathematical Programming Series B, 96(2) 293-320.

10. Prajna, S., Papachristodoulou, A., Seiler, P., Parrilo, P.A. (2004) SOS-TOOLS: Sum of squares optimization toolbox for MATLAB.

11. Primbs, J.A. (2008) Optimization based option pricing bounds via piecewise polynomial super- and sub-martingales, In: 2008 American Control Conference.

12. Protter, P. Talay, D. (1997) The Euler scheme for Lévy driven stochastic differential equations, Annals of Probability, 25, 393-423.

13. Sato, K. (1999) Lévy processes and infinitely divisible distributions, Cambridge University Press.

14. Sturm, J. (2006) SeDuMi version 1.1. 